\title{
A Gallery of Synchronization Phenomena in Resistively Coupled Non-autonomous Chaotic Circuits
}

\author{
Ch. K. Volos ${ }^{*}, 1$, I. M. Kyprianidis ${ }^{2}$ and I. N. Stouboulos ${ }^{2}$ \\ ${ }^{I}$ Faculty of Mathematics and Engineering Studies, Dept. of Military Science, Hellenic Army Academy, Vari, GR-16673, Greece \\ ${ }^{2}$ Physics Department, Aristotle University of Thessaloniki, GR-54124, Greece
}

Received 2 June 2013; Revised 4 September 2013; Accepted 25 September 2013

\begin{abstract}
This work deals with the study of a variety of synchronization phenomena in the case of resistively coupled nonautonomous, nonlinear circuits. In this paper, a very simple but very representative second order, non-autonomous, nonlinear circuit, is used, the Lacy circuit. Also, two different approaches of coupling between such circuits are applied. The first one is the well-known mutual coupling via a linear resistor, in which the phenomena of complete and anti-phase synchronization are observed and explained based on the nature of this kind of nonlinear systems. The second one is a ring connection in a neural-type system, where the Lacy acts as the master circuit. In this case a very interesting type of partial synchronization, between the other two circuits of this topology, is presented for the first time.
\end{abstract}

Keywords: Nonlinear system, Lacy circuit, chaos, mutual coupling, ring connection, anti-phase synchronization, complete chaotic synchronization, partial synchronization.

\section{Introduction}

In the last thirty years the interaction between coupled chaotic systems became a topic of great interest due to the applications not only in a variety of complex physical, chemical and biological systems [1-2] but also in many interesting commercial activities such as secure communications and cryptography [3-4]. Nevertheless, the cornerstone of this research topic was the introduction of the synchronization's theory in 1980's and early 1990's [5-7].

Since then, various types of synchronization, depending of the coupling scheme and the nature of the interacting systems have been reported. Phase synchronization, Lag synchronization, Generalized synchronization, Projective synchronization, Anticipating synchronization, Inverse lag synchronization and Inverse $\pi$-lag synchronization [8-15] are some of the most interesting types of synchronization, which have been investigated thoroughly until now.

However, the most interesting and the most studied case of synchronization is the Complete or Full synchronization $[5,6,16,17]$. In this case the interaction between two coupled identical nonlinear circuits leads to a perfect coincidence of their chaotic trajectories, i.e.

$x_{1}(t)=x_{2}(t)$, as $t \rightarrow \infty$.

\footnotetext{
*E-mail address: chvolos@gmail.com

ISSN: 1791-2377 (C) 2013 Kavala Institute of Technology. All rights reserved.
}

Also, in 1998, Cao and Lai observed an interesting type of synchronization between mutually coupled identical autonomous nonlinear systems [18]. In this new type of synchronization, which is called Anti-phase synchronization, each one of the uncoupled systems produces chaotic attractors. Also, the equations governing the coupled systems must be symmetrical with respect to the origin, i.e. they are invariant under the transformation:

$\mathrm{S}:(x, y, z) \rightarrow(-x,-y,-z)$

Furthermore, this synchronization phenomenon is observed when the coupled system is in a phase locked (periodic) state, depending on the coupling factor and it can be characterized by a $\pi$-phase delay. So, the periodic signals $\left(x_{1}\right.$ and $\left.x_{2}\right)$ of each coupled circuits have a time lag $\tau$, which is equal to $T / 2$, where $T$ is the period of the signals $x_{1}$ and $x_{2}$.

$x_{1}(t)=x_{2}(t+\tau), \quad \tau=\mathrm{T} / 2$

The anti-phase synchronization was also observed by Volos et al. in the case of two mutually coupled identical non-autonomous Duffing-type systems [14], which as it is known, have symmetry, because the transformation:

$\mathrm{S}:(x, y, t) \rightarrow(-x,-y, t+\mathrm{T} / 2)$

leaves Duffing's system equations invariant. 
In the present paper, the study of various synchronization phenomena between resistively coupled non-autonomous, nonlinear circuits is presented. For this reason, the wellknown Lacy circuit, which belongs to the family of nonautonomous circuits that obey the symmetry (4), is used. So, in the case of two mutually coupled Lacy circuits, except of the complete chaotic synchronization, the existence of antiphase synchronization is also confirmed from the simulation results.

However, the most interesting phenomenon and a totally new case of synchronization in a ring connection between the resistively coupled Lacy circuit with two other autonomous nonlinear circuits of this family, is observed. This new case of synchronization, which is a specific type of partial synchronization, is due to the topology of the neuraltype system, which is adopted.

This paper is organized as follows. In Section 2, the nonlinear circuit, the Lacy circuit, which is used in this work, is presented in details. Section 3 describes the mutual coupling scheme of two identical Lacy circuits, while the simulation results confirm the observation of the anti-phase synchronization phenomenon. In Section 4, a neural-type system in ring connection, based on the Lacy circuit, is described. Also, the study of this new type of partial synchronization between the circuits of this system is presented in the same Section. Finally, Section 5 includes the conclusions of this work.

\section{The Lacy Circuit}

In this work the Lacy circuit (Fig.1), which is a second order nonlinear, non-autonomous circuit, is used [19]. The state equations of the Lacy circuit in normalized form are given by:

$$
\left\{\begin{array}{l}
\frac{\mathrm{d} x}{\mathrm{~d} \tau}=y-f(x) \\
\frac{\mathrm{d} y}{\mathrm{~d} \tau}=-\gamma \cdot(x+y)+\mathrm{V}_{\mathrm{N}} \cdot \sin \Omega \tau
\end{array}\right.
$$

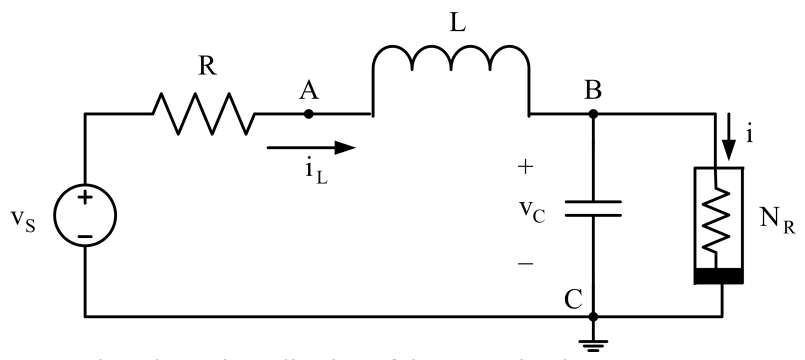

Fig. 1. The schematic realization of the Lacy circuit.

where, $f(x)$ is the nonlinear element $\left(\mathrm{N}_{\mathrm{R}}\right)$ of this circuit, which is a piecewise linear resistor that has a $v-i$ characteristic of N-type (Fig. 2). In this figure $\mathrm{P}_{-}, \mathrm{O}$ and $\mathrm{P}_{+}$ are the equilibrium points of the circuit. $\mathrm{P}_{-}$and $\mathrm{P}_{+}$are stable, while $\mathrm{O}$ is unstable. The slope of the load line is equal to $1 / \mathrm{R}$. This nonlinear element is described by the following equation.

$$
f(x)=\mathrm{m}_{\mathrm{b}} \cdot x+0.5 \cdot\left(\mathrm{m}_{\mathrm{a}}-\mathrm{m}_{\mathrm{b}}\right) \cdot\{|x+1|-|x-1|\}
$$

The device implementing such a characteristic is shown in Fig.3.

In this paper, in contrary to other similar works, an inductorless implementation of the Lacy circuit is used. The principle to which such implementation is based, is to substitute the inductor with an equivalent circuit based on operational amplifiers [20]. The realization of this circuit is shown in Fig.4, where it implements an equivalent inductor with the following value:

$$
\mathrm{L}=\frac{\mathrm{R}_{\mathrm{L} 1} \mathrm{R}_{\mathrm{L} 3} \mathrm{R}_{\mathrm{L} 4} \mathrm{C}_{\mathrm{L}}}{\mathrm{R}_{\mathrm{L} 2}}
$$

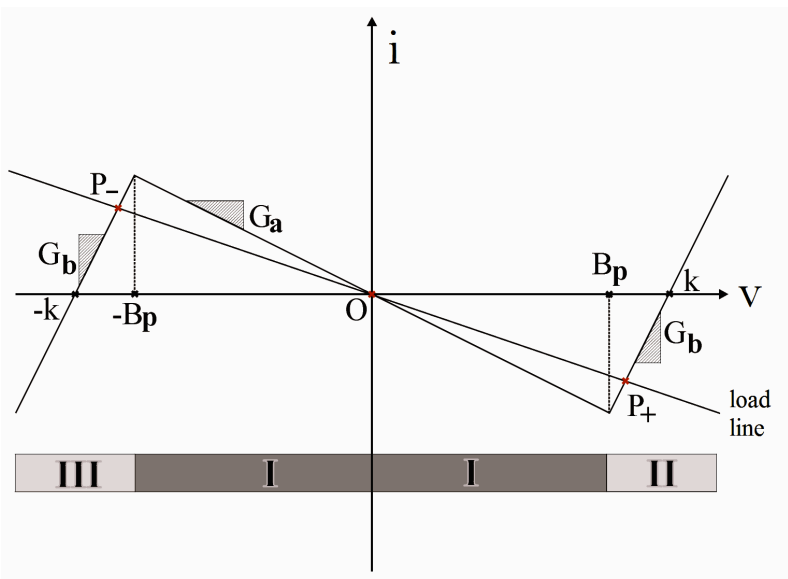

Fig. 2. The v-i characteristic of the nonlinear resistor of the circuit of Fig.1.

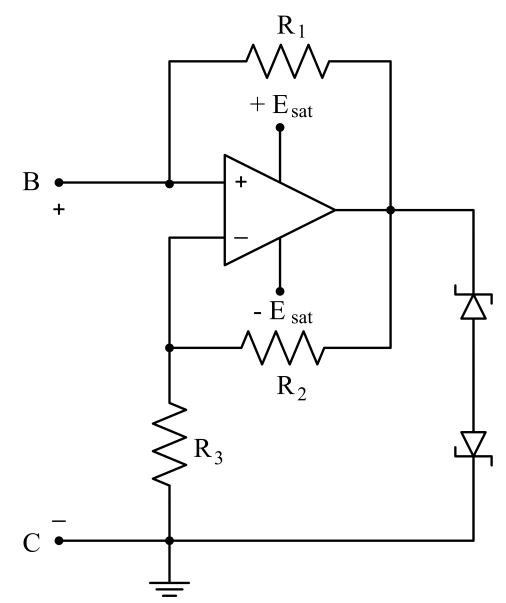

Fig. 3. Electronic device for the implementation of the $v-i$ characteristic of Eq.(6).

In system's equations (5) and (6), $x_{1}=\mathrm{v}_{\mathrm{Cl}} / \mathrm{B}_{\mathrm{p}}$, $x_{2}=\mathrm{v}_{\mathrm{C} 2} / \mathrm{B}_{\mathrm{p}}, y_{1}=\mathrm{Ri}_{\mathrm{L} 1} / \mathrm{B}_{\mathrm{p}}, y_{2}=\mathrm{Ri}_{\mathrm{L} 2} / \mathrm{B}_{\mathrm{p}}, \tau=t / \mathrm{RC}, \Omega=\omega \mathrm{RC}$, $\gamma=\mathrm{R}^{2} \mathrm{C} / \mathrm{L}, \mathrm{V}_{\mathrm{N}}=\gamma \mathrm{V}_{0} / \mathrm{B}_{\mathrm{p}}, \mathrm{G}_{\mathrm{a}}=-1 / \mathrm{R}_{3}, \mathrm{G}_{\mathrm{b}}=1 / \mathrm{R}_{1}, \mathrm{~m}_{\mathrm{a}}=\mathrm{RG}_{\mathrm{a}}$, and $\mathrm{m}_{\mathrm{b}}=\mathrm{RG}_{\mathrm{b}}$, are the normalized variables and the system's parameters. Also, the parameter's values of the circuit, which are used in this work, are: $\mathrm{R}=0.7 \mathrm{k} \Omega, \mathrm{C}=62.9 \mathrm{nF}$, $\mathrm{G}_{\mathrm{a}}=-2.2 \mathrm{mS}, \mathrm{G}_{\mathrm{b}}=1.0 \mathrm{mS}, \mathrm{Bp}=1.878 \mathrm{~V}, \mathrm{R}_{1}=\mathrm{R}_{3}=1 \mathrm{k} \Omega$, $\mathrm{R}_{2}=2.2 \mathrm{k} \Omega$ and $\mathrm{L}=32.9 \mathrm{mH}$, (where, $\mathrm{R}_{\mathrm{L} 1}=\mathrm{R}_{\mathrm{L} 2}=\mathrm{R}_{\mathrm{L} 3}=$ $\left.1 \mathrm{k} \Omega, \mathrm{R}_{\mathrm{L} 4}=32.9 \mathrm{k} \Omega, \mathrm{C}_{\mathrm{L}}=1 \mathrm{nF}\right)$. The two back-to-back zener diodes in Fig. 3 have $\mathrm{E}_{\mathrm{z}}=6 \mathrm{~V}$, while the op-amp is of type LF411 with $\mathrm{E}_{\mathrm{sat}}= \pm 9 \mathrm{~V}$. Furthermore, $\mathrm{V}_{0}$ is the amplitude of the sinusoidal voltage source, and $\mathrm{f}=\omega / 2 \pi$, its frequency. 


\section{The Mutual Coupling Scheme}

Since nonlinear systems and especially chaotic systems exhibit high sensitivity on initial conditions and thus, if they are identical and, possibly, starting from almost the same initial points, following trajectories which rapidly become uncorrelated, appropriate techniques should be set up to obtain synchronization. Such techniques to couple two or more chaotic systems can be mainly divided into two classes: drive-response or unidirectional coupling and bidirectional or mutual coupling [21]. In the first case, one system drives another one called the response or slave system, while on the contrary, in mutual coupling both the circuits are connected and each circuit influences the dynamics of the other. So, the mutually coupled systems show always more complex dynamic behavior. The case of bidirectional coupling between two coupled chaotic oscillators is described by the following set of differential equations:

$$
\left\{\begin{array}{l}
\dot{x}_{1}=F\left(x_{1}\right)+C \cdot\left(x_{2}-x_{1}\right) \\
\dot{x}_{2}=G\left(x_{2}\right)+C \cdot\left(x_{1}-x_{2}\right)
\end{array}\right.
$$

while in the case of unidirectional coupling the system of differential equation is written as:

$$
\left\{\begin{array}{l}
\dot{x}_{1}=F\left(x_{1}\right) \\
\dot{x}_{2}=F\left(x_{2}\right)+C \cdot\left(x_{1}-x_{2}\right)
\end{array}\right.
$$

where $F(x)$ are vector fields in the phase space of dimension n, i.e. $x \in \mathbb{R}^{\mathrm{n}}$, and $C$ is a symmetric matrix of constants which describes the nature and strength of the coupling between the oscillators.

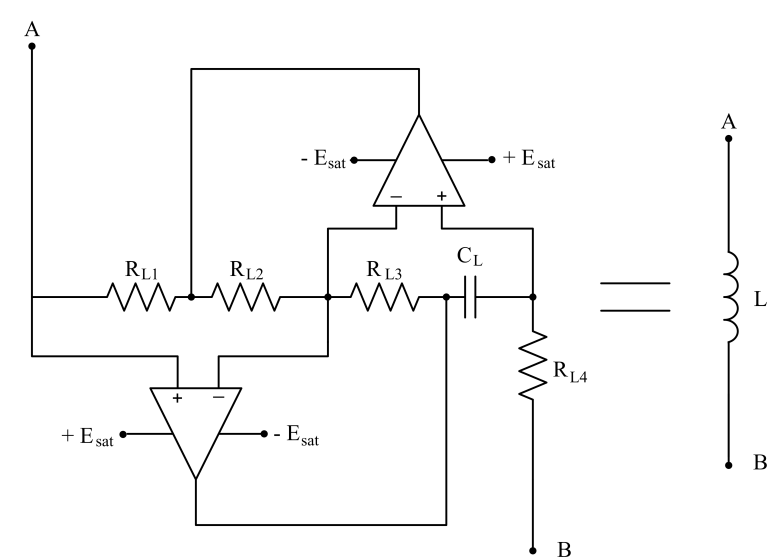

Fig. 4. Equivalent circuit of an inductor based on operational amplifiers.

\subsection{System's Description}

In this work the mutual or bidirectional coupling, between two identical Lacy circuits, is achieved via a linear resistor $\mathrm{R}_{\mathrm{C}}$ connected between the nodes $\mathrm{A}$ of each circuit (Fig.5). The dimensionless system of differential equations that describes the coupled system's dynamics is:

$$
\left\{\begin{array}{l}
\frac{\mathrm{d} x_{1}}{\mathrm{~d} \tau}=y_{1}-f\left(x_{1}\right)+\xi \cdot\left(x_{2}-x_{1}\right) \\
\frac{\mathrm{d} y_{1}}{\mathrm{~d} \tau}=-\gamma \cdot\left(x_{1}+y_{1}\right)+\mathrm{V}_{\mathrm{N}} \cdot \sin \Omega \tau \\
\frac{\mathrm{d} x_{2}}{\mathrm{~d} \tau}=y_{2}-f\left(x_{2}\right)+\xi \cdot\left(x_{1}-x_{2}\right) \\
\frac{\mathrm{d} y_{2}}{\mathrm{~d} \tau}=-\gamma \cdot\left(x_{2}+y_{2}\right)+\mathrm{V}_{\mathrm{N}} \cdot \sin \Omega \tau
\end{array}\right.
$$

The first two equations of system (10) describe the first of the two coupled identical Lacy circuits while the other two describe the second one. Also, the parameter $\xi=\mathrm{R} / \mathrm{R}_{\mathrm{C}}$ is the coupling coefficient and it is present in the equations of both circuits, since the coupling between them is mutual.

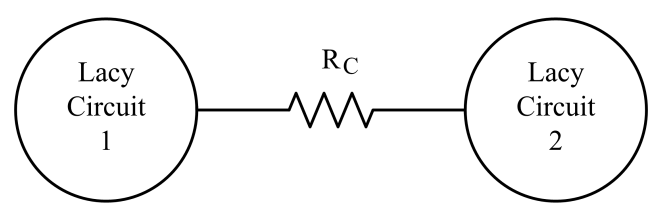

Fig. 5. Two bidirectionally or mutually coupled Lacy circuits via a linear resistor.

\subsection{Simulation Results}

In this Section, the dynamic behavior of two mutually coupled Lacy circuits is investigated numerically by employing a fourth order Runge-Kutta algorithm. So, by solving the coupled system's equations (11) the bifurcation diagram of the signal's difference $\left(x_{2}-x_{1}\right)$ versus the coupling factor $\xi$ is produced. In details, this diagram is produced by increasing the coupling factor $\xi$, from $\xi=0$ (uncoupled system) to $\xi=0.2$ with step $\Delta \xi=0.0004$, while initial conditions in each iteration have different values. This occurs because the last values of the state variables in the previous iteration become the initial values for the next iteration. This type of bifurcation diagram is more close to the experimental observation of the system's dynamic behavior as the coupling factor $\xi$ is increased by using a variable resistor for $\mathrm{R}_{\mathrm{C}}$.

So, the bifurcation diagram of Fig. 6 is produced by using the previous mentioned circuit's parameters, for $\mathrm{f}=7 \mathrm{kHz}$ and $\mathrm{V}_{0}=5.5 \mathrm{~V}$, while the system's initial conditions are: $\left(x_{10}, y_{10}, x_{20}, y_{20}\right)=(0.5,0.1,-0.4,-0.2)$.

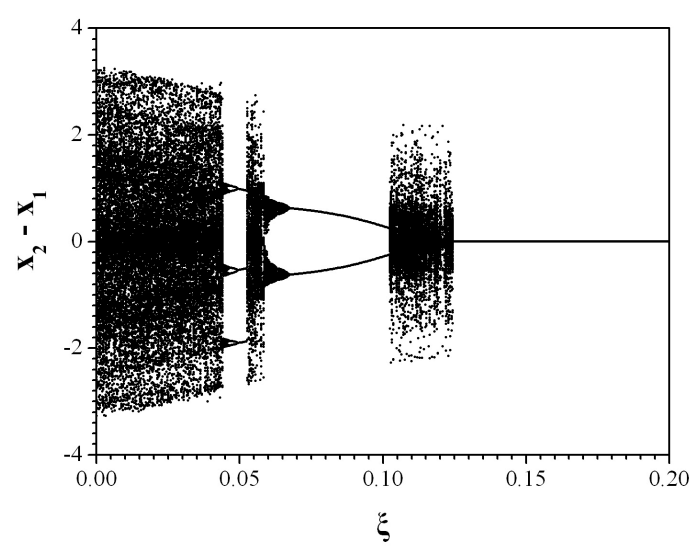

Fig. 6. Bifurcation diagrams of $\left(x_{2}-x_{1}\right)$ versus $\xi$, for $\mathrm{f}=7 \mathrm{kHz}$ and $\mathrm{V}_{0}=5.5 \mathrm{~V}$. 
This bifurcation diagram shows that the coupled system undergoes from full desynchronization, for $\xi<$ 0.046 , where each circuit is in a chaotic state and lays on its own manifold (Fig.7), to complete chaotic synchronization (Fig.8), for $\xi \geq 0.125$, where their manifolds coincide, through an intermediate region where the system shows a more complex dynamic behavior. This is a typical transition from full desynchronization to complete synchronization.

The intermediate region of the bifurcation diagram of Fig. 6 is more complicated and it can be divided in four discrete regions:

- Region I: $0.046<\xi \leq 0.053$ (Period-3 steady state).

- Region II: $0.053<\xi \leq 0.067$ (Chaotic desynchronization).

- Region III: $0.067<\xi \leq 0.100$ (Period-2 steady state).

- Region IV: $0.100<\xi \leq 0.125$ (Chaotic desynchronization).

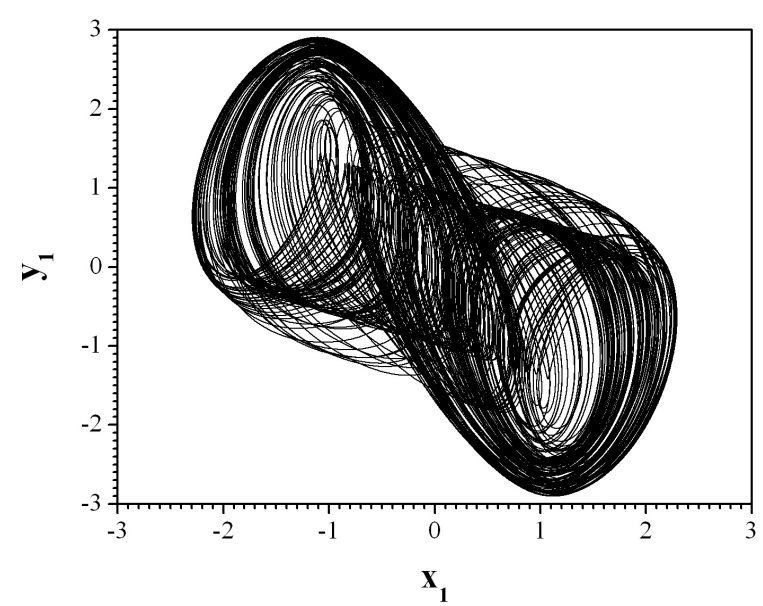

Fig. 7. Simulation phase portrait of $y_{1}$ vs. $x_{1}$ for $\xi=0.02$ (chaotic double-scroll attractor).

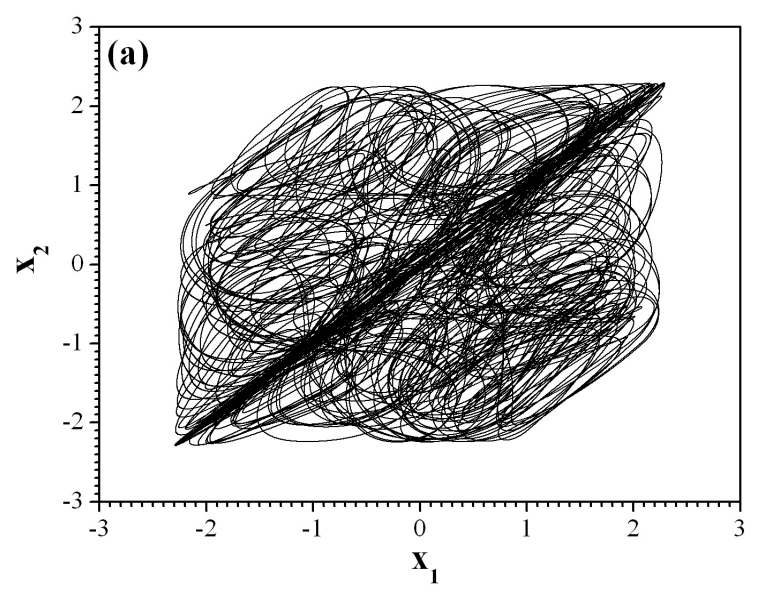

Fig. 8. Simulation phase portrait of $x_{2}$ vs. $x_{1}$ for $\xi=0.15$ (chaotic synchronization state).

However, in this work we have focused on Region III of the intermediate region, where the coupled system shows the phenomenon of anti-phase synchronization.

In this region the system has periodic behavior and specifically shows a period-2 steady state. This occurs because each one of the coupled circuits remains in the same periodic state. Figures 9(a) and 9(b) show the simulation phase portraits of $x_{2}$ versus $x_{1}$ and $y_{1,2}$ versus $x_{1,2}$, for $\xi=0.08$, respectively. In the first of these figures the periodic behavior (period-2) of the coupled system is observed, while in the second one the coincidence of circuits' attractors in the phase plain is presented.

Furthermore, in Fig.10, the time-series of the state variables $x_{1}$ and $x_{2}$ of the coupled circuits are shown. It is obvious that the two signals $x_{1}$ and $x_{2}$ are identical with a time lag.
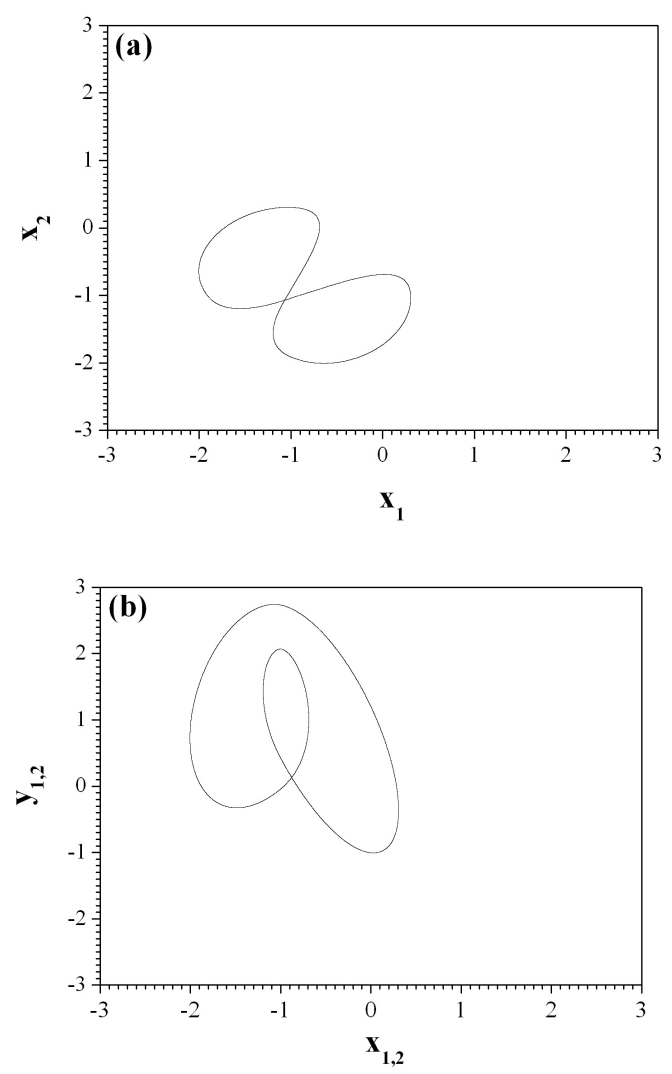

Fig. 9. Simulation phase portrait of (a) $x_{2}$ vs. $x_{1}$ and (b) $y_{1,2}$ vs. $x_{1,2}$, for $\xi=0.08$ (anti-phase synchronization).

To quantify this time lag we have used the well-known Similarity function $S$ [9], defined with respect to the state variables $x_{1}$ and $x_{2}$.

$S(\tau)=\sqrt{\frac{\left\langle\left[x_{2}(t+\tau)-x_{1}(t)\right]^{2}\right\rangle}{\left[\left\langle\left(x_{1}(t)\right)^{2}\right\rangle \cdot\left\langle\left(x_{2}(t)\right)^{2}\right\rangle\right]^{1 / 2}}}$

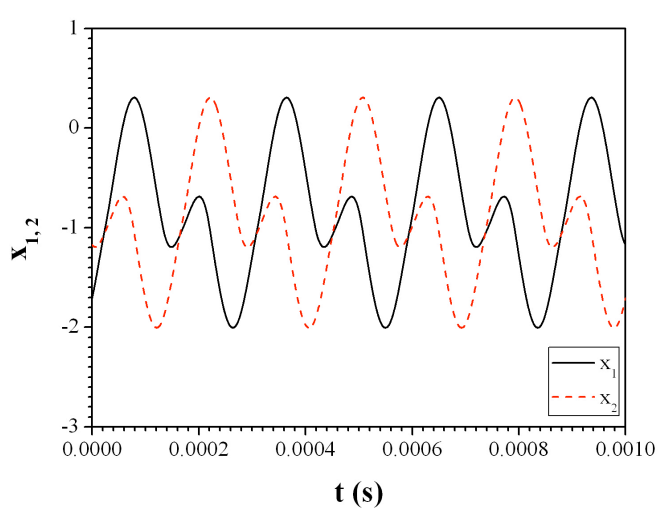

Fig. 10. Time-series of $x_{1}$ and $x_{2}$, for $\xi=0.08$. 
Let $S_{\min }$ be the minimum value of the Similarity function $S(\tau)$ and let $\tau_{\min }$ be the amount of time lag, when $S_{\min }$ is achieved. The time lag $\tau_{\min }$ between the variables $x_{1}$ and $x_{2}$ is found, when the conditions $S_{\min }=0$ and $\tau_{\min } \neq 0$ are fulfilled. The calculation of the similarity function for $\xi=0.08$ (Fig.11) shows that the expected time lag $\tau_{\min }=0.1429 \mathrm{~ms}$, is equal to $\mathrm{T} / 2$, where $\mathrm{T}$ is the period of $x_{1}$ and $x_{2}$.

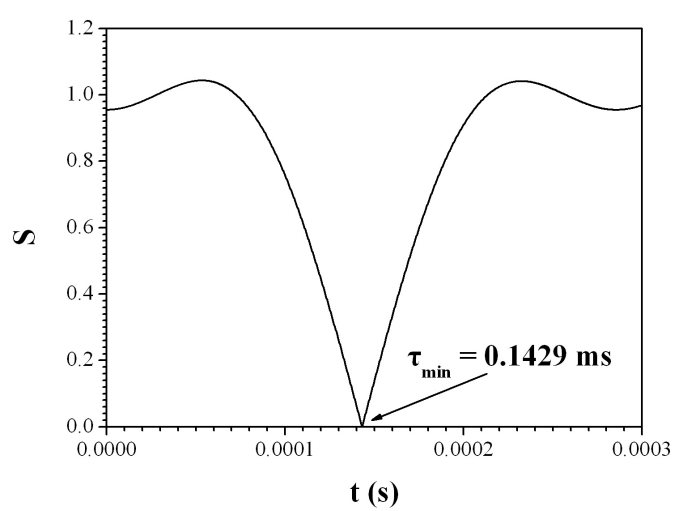

Fig. 11. The similarity function $(S)$ versus time $(t)$, for $\xi=0.08$. $S_{\min }=0$ means lag with time shift of $\tau_{\min }=0.1429 \mathrm{~ms}=\mathrm{T} / 2$. So, the phenomenon of anti-phase synchronization is confirmed.

Furthermore, the same time lag is found for every value of coupling factor $(\xi)$ in the Region III. So, the value of time lag remains always the same in this region and equals to the half of the period of the external voltage source.

Moreover the fact that the difference of $\left[x_{1}(t)-x_{2}(t+\mathrm{T} / 2)\right]$ is equal to zero (Fig.12), confirms that the coupled system demonstrates $\pi$ phase delay, which is defined as anti-phase synchronization or $\pi$-lag synchronization.

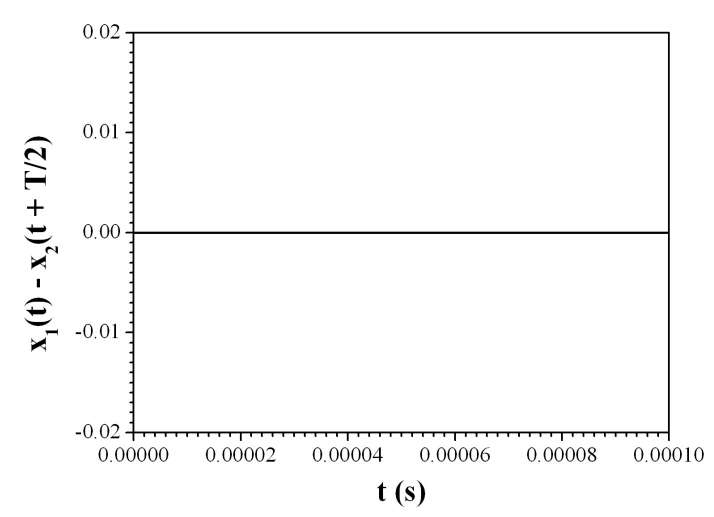

Fig. 12. Time-series of $x_{1}(t)-x_{2}(t+\mathrm{T} / 2)$, for $\xi=0.08$.

\section{Dynamics of a Neural-type System in Ring Topology}

In this Section, the nonlinear and non-autonomous Lacy circuit is connected as a master circuit through the node "A1" to the nonlinear autonomous circuits "2" and "3" via buffers in a ring topology (Fig.13). This topology has many similarities with neural-type systems, in which the master circuit acts as a pre-synaptic neuron that receives the external stimulation and then transmits a signal (information) to the other post-synaptic neurons (autonomous circuits "2" and "3") of the system. So, in this case the dynamic state of the master circuit remains unaffected, while the states of the two other circuits are influenced by the dynamics of its master. For this reason, the dynamic state of the master circuit is chosen to be chaotic and the responses of the two other circuits are studied via the simulation results.

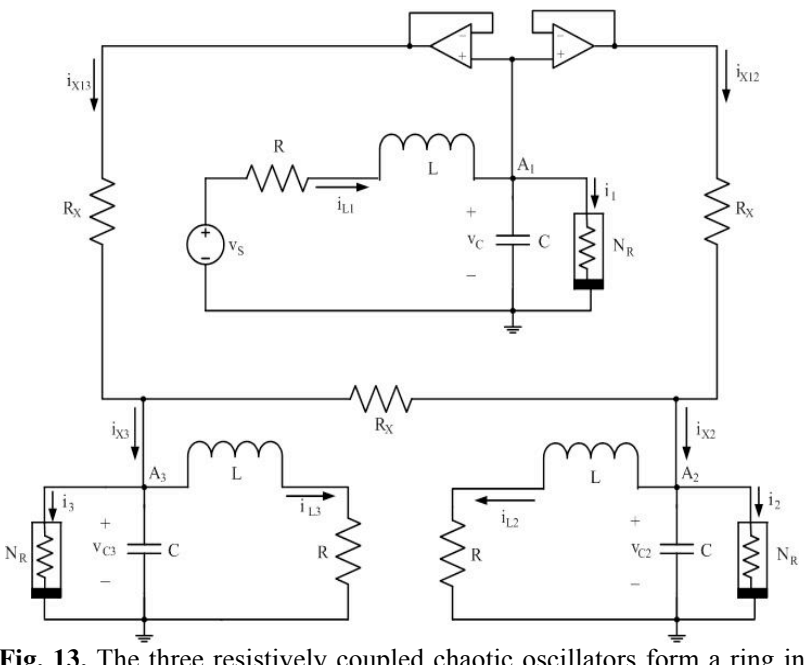

Fig. 13. The three resistively coupled chaotic oscillators form a ring in neural-type connection.

\subsection{System's Description}

The normalized state equations of the proposed system are the following:

$\left\{\begin{array}{l}\frac{\mathrm{d} x_{1}}{\mathrm{~d} \tau}=y_{1}-f\left(x_{1}\right) \\ \frac{\mathrm{d} y_{1}}{\mathrm{~d} \tau}=-\gamma \cdot\left(x_{1}+y_{1}\right)+\mathrm{V}_{\mathrm{N}} \cdot \sin \Omega \tau \\ \frac{\mathrm{d} x_{2}}{\mathrm{~d} \tau}=y_{2}-f\left(x_{2}\right)+\xi \cdot\left(x_{1}-2 x_{2}+x_{3}\right) \\ \frac{\mathrm{d} y_{2}}{\mathrm{~d} \tau}=-\gamma \cdot\left(x_{2}+y_{2}\right) \\ \frac{\mathrm{d} x_{3}}{\mathrm{~d} \tau}=y_{3}-f\left(x_{3}\right)+\xi \cdot\left(x_{1}+x_{2}-2 x_{3}\right) \\ \frac{\mathrm{d} y_{3}}{\mathrm{~d} \tau}=-\gamma \cdot\left(x_{3}+y_{3}\right)\end{array}\right.$

where

$$
f\left(\mathrm{x}_{1,2,3}\right)=\mathrm{m}_{\mathrm{b}} \mathrm{x}_{1,2,3}+0.5\left(\mathrm{~m}_{\mathrm{a}}-\mathrm{m}_{\mathrm{b}}\right)\left\{\left|\mathrm{x}_{1,2,3}+1\right|-\left|\mathrm{x}_{1,2,3}-1\right|\right\}
$$

is the normalized characteristic function of the nonlinear resistors, according to Eq.(6). The first two equations of system (13) describe the master circuit, while the other four equations, in couples, describe the other two nonlinear autonomous circuits of the proposed system.

\subsection{Simulation Results}

Next, the dynamic behavior of the system of Fig.13 was studied. For low values of the coupling factor $\xi(\xi<0.9)$, 
two different dynamic behaviors have been observed. These different dynamics are related to the initial conditions of the state variables of the system as it will be shown in details.

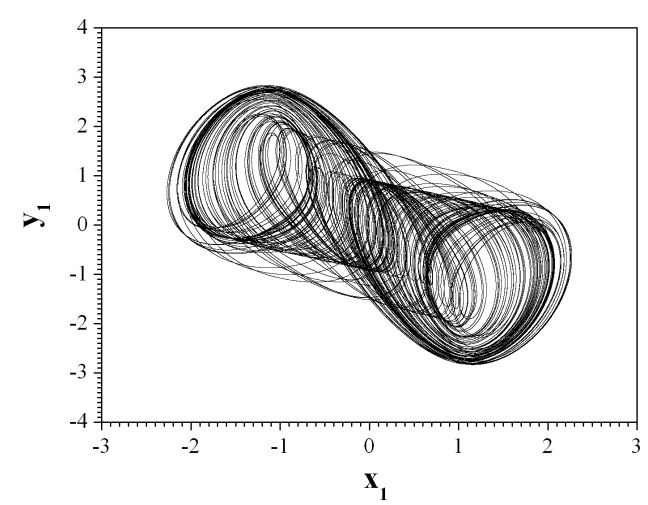

Fig. 14. Phase portrait of circuit " 1 ", for $\mathrm{f}=7 \mathrm{kHz}$ and $\mathrm{V}_{0}=5.35 \mathrm{~V}$.

\subsubsection{Different Basins of Attraction}

As it is mentioned, the dynamics of the non-autonomous circuit " 1 " is not affected by the dynamics of the autonomous circuits " 2 " and " 3 ", because of the unidirectional coupling. The initial conditions of the state variables of the system are: $\left(x_{10}, y_{10}, x_{20}, y_{20}, x_{30}, y_{30}\right)=$ $(1.00,1.25,-1.20,1.50,0.80,-1.00)$. The initial conditions of the state variables of circuit " 2 " belong to the basin of attraction of the equilibrium point $\mathrm{P}$, while the initial conditions of the state variables of circuit " 3 " belong to the basin of attraction of the equilibrium point $\mathrm{P}_{+}$. For $\mathrm{f}=7 \mathrm{kHz}$ and $\mathrm{V}_{0}=5.35 \mathrm{~V}$ circuit " 1 " is in a chaotic state (Fig.14). Choosing the coupling factor $\xi=0.010$, the autonomous circuits are also in chaotic states, as it can be shown in Figs.15(a) \& (b).
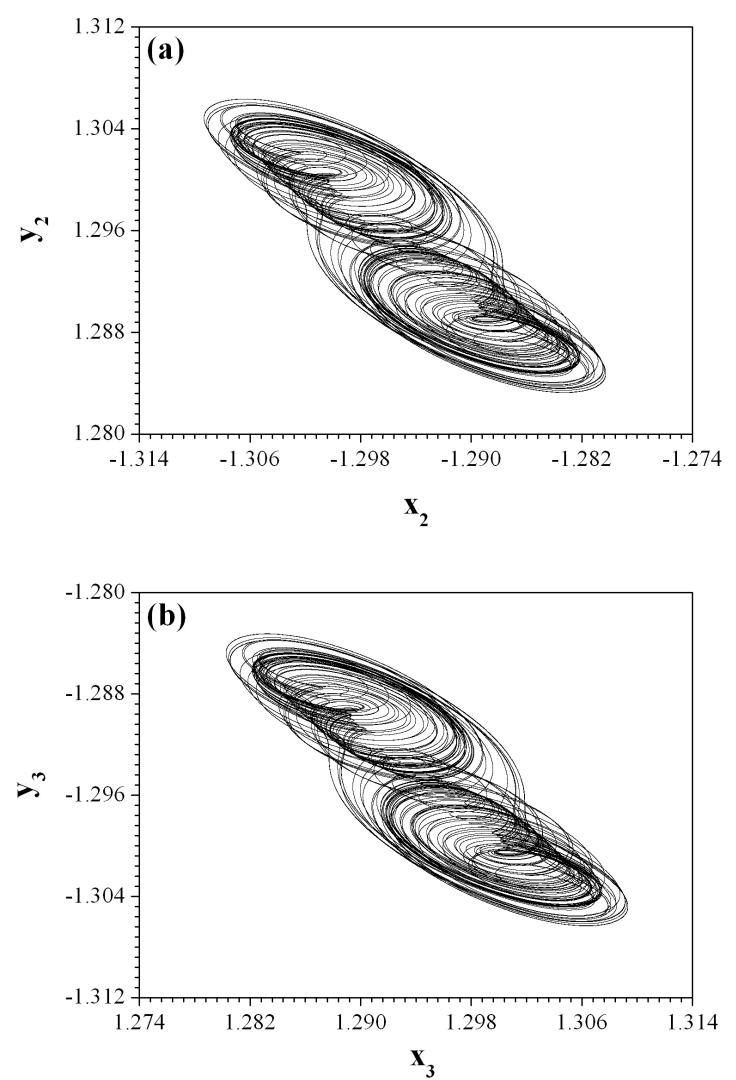

Fig. 15. (a) \& (b) Phase portraits of the state variables of circuits "2" and " 3 ", for $\mathrm{f}=7 \mathrm{kHz}, \mathrm{V}_{0}=5.35$ and $\xi=0.010$, for initial conditions belonging to different basins of attraction.
In order to study the correlation among the state variables of the system, the phase portraits of Fig.16 have been plotted. Phase portraits of Figs.16(e) \& (f) do not correspond to synchronization or antisynchronization between the state variables of the autonomous circuits " 2 " and " 3 ". The fact, that the slope of straight lines are equal to unity, means that $\Delta x_{2}=\Delta x_{3}$ and $\Delta y_{2}=\Delta y_{3}$, while taking the limit we have $\mathrm{d} x_{2}=\mathrm{d} x_{3}$ and $\mathrm{d} y_{2}=\mathrm{d} y_{3}$ or

$$
\begin{aligned}
& \frac{d x_{2}}{d \tau}=\frac{d x_{3}}{d \tau} \\
& \text { and } \\
& \frac{d y_{2}}{d \tau}=\frac{d y_{3}}{d \tau}
\end{aligned}
$$

because they are dynamic state variables.
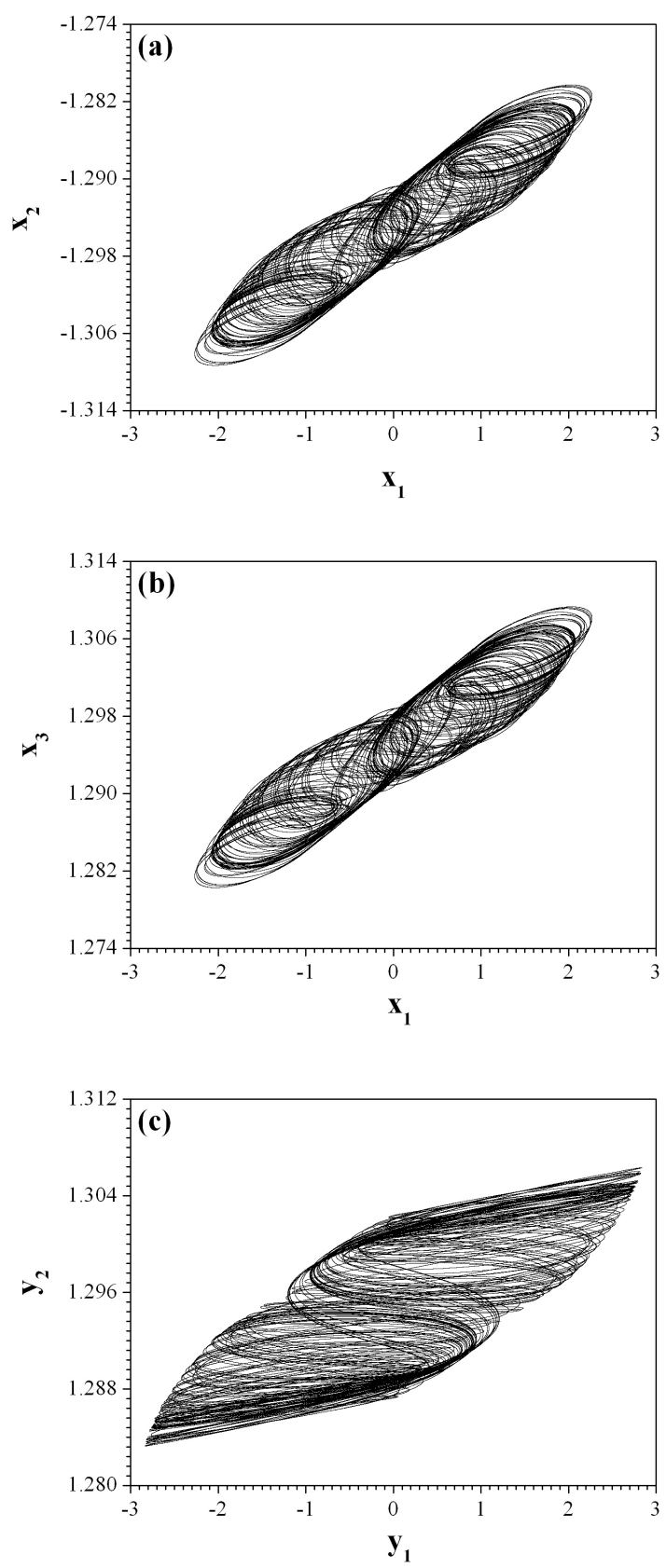

(continued) 

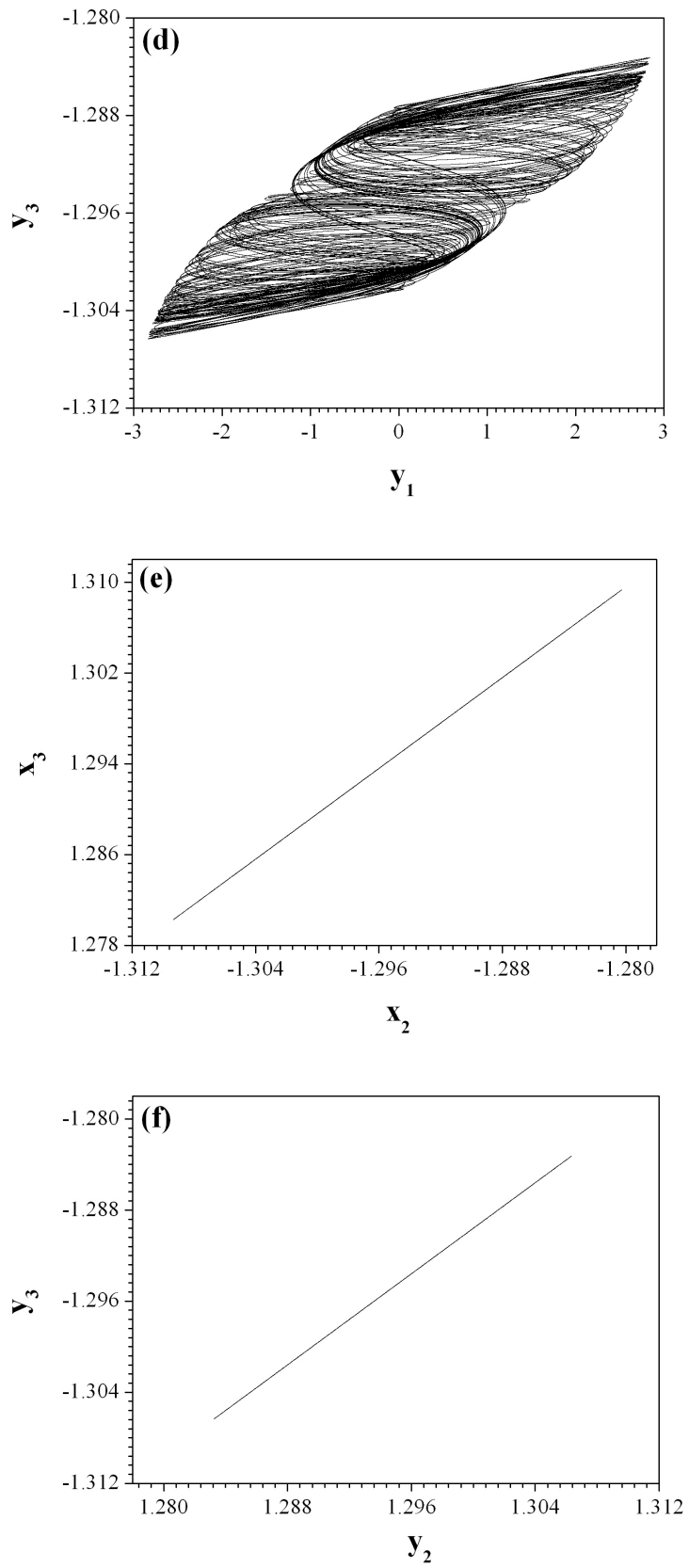

Fig. 16. Phase portraits of (a) $x_{2}$ vs. $x_{1}$, (b) $x_{3}$ vs. $x_{1}$, (c) $y_{2}$ vs. $y_{1}$, (d) $y_{3}$ vs. $y_{1}$, (e) $x_{3}$ vs. $x_{2}$ and (f) $y_{3}$ vs. $y_{2}$, for $\mathrm{f}=7 \mathrm{kHz}, \mathrm{V}_{0}=5.35$ and $\xi=0.010$.

As it is mentioned before, $x_{2}=\mathrm{v}_{\mathrm{C} 2} / \mathrm{B}_{\mathrm{p}}, x_{3}=\mathrm{v}_{\mathrm{C} 3} / \mathrm{B}_{\mathrm{p}}$, and replacing in Eq.(15) the normalized variables, we obtain $\mathrm{dv}_{\mathrm{C} 2} / \mathrm{d} t=\mathrm{dv}_{\mathrm{C} 3} / \mathrm{d} t$, or

$\mathrm{C} \frac{\mathrm{dv}_{\mathrm{C} 2}}{\mathrm{~d} t}=\mathrm{C} \frac{\mathrm{dv}_{\mathrm{C} 3}}{\mathrm{~d} t}$

The result of Eq.(17) means that $\mathrm{i}_{\mathrm{C} 2}=\mathrm{i}_{\mathrm{C} 3}$, so the currents through the capacitors of the circuits "2" and " 3 " are complete synchronized.
Also, $y_{2}=\mathrm{Ri}_{\mathrm{L} 2} / \mathrm{B}_{\mathrm{P}}$ and $y_{3}=\mathrm{Ri}_{\mathrm{L} 3} / \mathrm{B}_{\mathrm{P}}$, and replacing in Eq.(16) we obtain $\mathrm{di}_{\mathrm{L} 2} / \mathrm{d} t=\mathrm{di}_{\mathrm{L} 3} / \mathrm{d} t$, or

$\mathrm{L} \frac{\mathrm{di}_{\mathrm{L} 2}}{\mathrm{~d} t}=\mathrm{L} \frac{\mathrm{di}_{\mathrm{L} 3}}{\mathrm{~d} t}$

The result of Eq.(18) means that $\mathrm{V}_{\mathrm{L} 2}=\mathrm{v}_{\mathrm{L} 3}$, so the voltages across the inductors of the circuits " 2 " and " 3 " are complete synchronized.

Combining the fourth and the sixth equation of system (13), as well as Eq.(16), the following equation is obtained.

$$
x_{2}+y_{2}=x_{3}+y_{3}
$$

This result is verified by the simulation of the system, as it is shown in Fig.17, where the phase portrait of $\left(x_{3}+y_{3}\right)$ versus $\left(x_{2}+y_{2}\right)$ has been plotted. In this case, only part of the corresponded electric variables of circuits " 2 " and " 3 " are synchronized to each other, while the state variables $\left(x_{2}, x_{3}\right)$ and $\left(y_{2}, y_{3}\right)$ corresponding to $\left(\mathrm{v}_{\mathrm{C} 2}, \mathrm{v}_{\mathrm{C} 3}\right)$ and $\left(\mathrm{i}_{\mathrm{L} 2}, \mathrm{i}_{\mathrm{L} 3}\right)$ respectively, remain unsynchronized. We have named this dynamics as partial synchronization of circuits " 2 " and " 3 ".

\subsubsection{Same Basins of Attraction}

In this section we have chosen the following initial conditions of system's state variables: $\left(x_{10}, y_{10}, x_{20}, y_{20}, x_{30}\right.$, $\left.y_{30}\right)=(1.00,1.25,-1.20,1.50,-0.80,1.00)$. In this case, the initial conditions of the state variables of circuit " 3 " belong to the basin of attraction of the equilibrium point $\mathrm{P}_{\text {-, as }}$, the initial conditions of the state variables of circuit "2". Circuits " 2 " and " 3 " are in a chaotic state (Fig.18) as the circuit " 1 " is (Fig.14). The phase portraits of $x_{3}$ versus $x_{2}$ and $y_{3}$ versus $y_{2}$, (Fig.19), reveal the complete synchronization between the waveforms of the voltages across the capacitors of circuits " 2 " and " 3 ", as well as between the waveforms of the currents through the inductors of the same circuits. In this case, because

$x_{2}=x_{3}$ and $y_{2}=y_{3}$

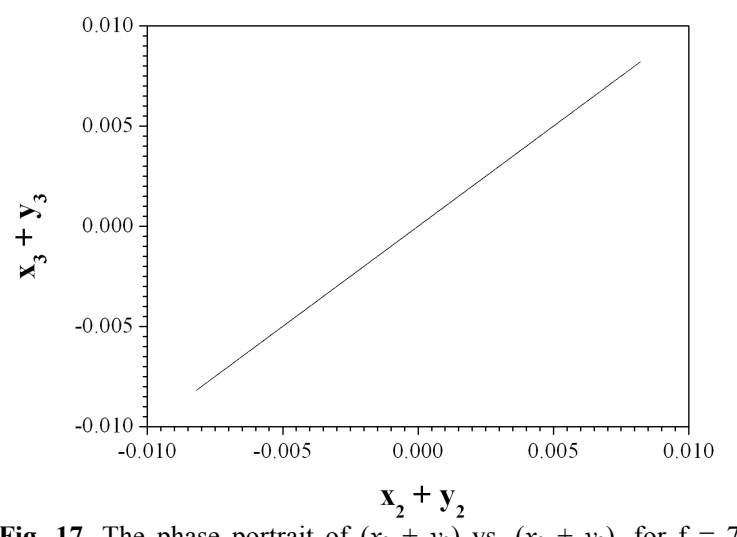

Fig. 17. The phase portrait of $\left(x_{3}+y_{3}\right)$ vs. $\left(x_{2}+y_{2}\right)$, for $\mathrm{f}=7 \mathrm{kHz}$, $\mathrm{V}_{0}=5.35$ and $\xi=0.010$. 

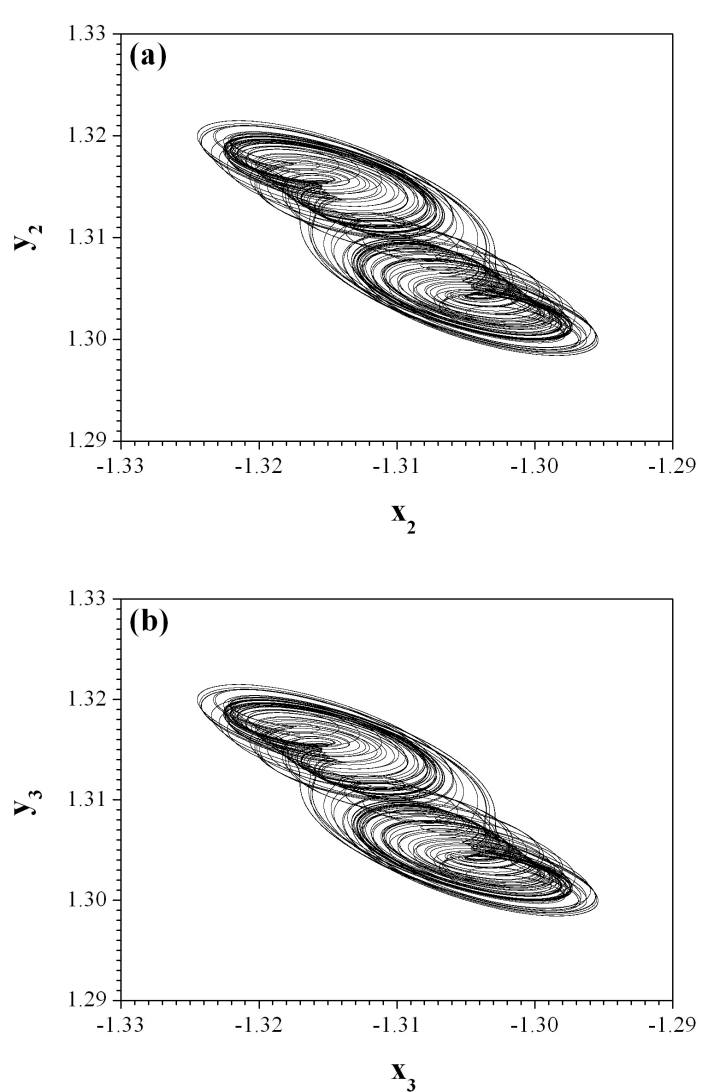

Fig. 18. (a) \& (b) Phase portraits of the state variables of circuits " 2 " \& "3", for $\mathrm{f}=7 \mathrm{kHz}, \mathrm{V}_{0}=5.35$ and $\xi=0.010$, for initial conditions belonging to the same basin of attraction.

Eq.(19) is also valid, so combining Eq.(20) with the fourth and sixth equation of system (13) we conclude that the voltages across the inductors of the circuits "2" and " 3 " are complete synchronized, while from Eq.(20) with the third and fifth equation of system (13), we also conclude that the currents through the capacitors of the circuits " 2 " and " 3 " are complete synchronized. So, all the corresponded electric variables of circuits " 2 " and " 3 " are synchronized to each other. We have named this dynamics complete synchronization of circuits " 2 " and " 3 ".

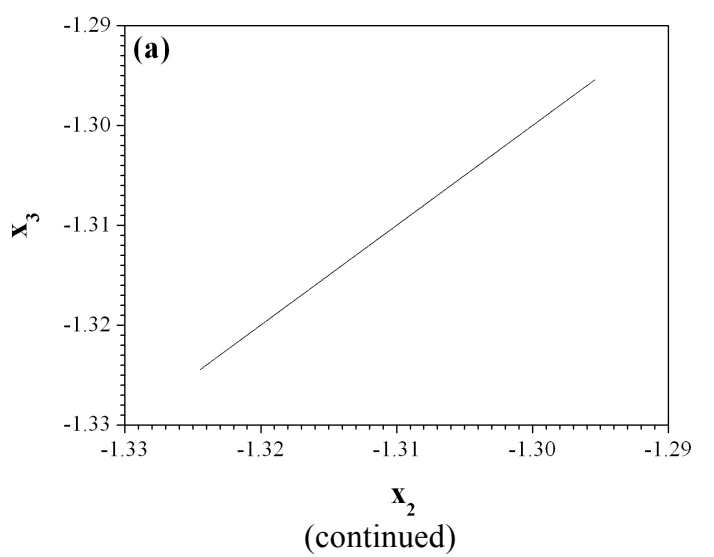

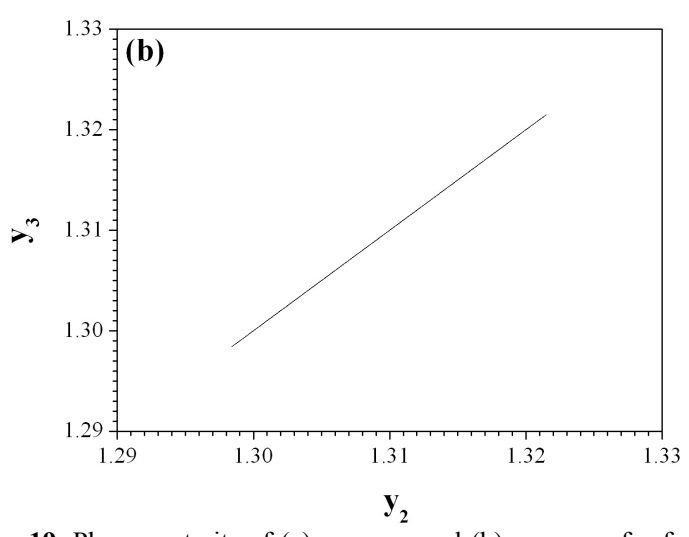

Fig. 19. Phase portraits of (a) $x_{3}$ vs. $x_{2}$ and (b) $y_{3}$ vs. $y_{2}$, for $\mathrm{f}=7 \mathrm{kHz}$, $\mathrm{V}_{0}=5.35$ and $\xi=0.010$, for initial conditions belonging to the same basin of attraction. The variables are in synchronization in both cases.

\section{Conclusion}

In the present paper, a gallery of various synchronization phenomena between resistively coupled nonlinear circuits, the Lacy circuits was presented. For this reason, two coupling schemes were adopted. The first one is the wellknown mutual coupling while the second one is a ring connection, like a neural-type system.

In the first case, two identical Lacy circuits have been coupled via a linear resistor and anti-phase synchronization was observed. In this type of synchronization the coupled circuits are in a periodic state and a $\pi$-phase delay was demonstrated. As it was mentioned, the state equations (5) of Lacy circuit's are invariant under the transformation (4). This fact, according to the conclusions of Ref.[14], leads to the observation that the coupled Lacy circuits have even mode (period-2) in the Region III and two conjugated periodic trajectories have arisen with a phase difference of $\pi$. Furthermore, the time lag remains always equal to the half of the period of the external voltage source for every value of the coupling factor in the region of anti-phase synchronization.

In the second case, a neural-type system in ring topology, in which the Lacy plays the role of a stimulated neuron-cell, while two other autonomous nonlinear circuits of this family were coupled via a linear resistor with it, was studied. As a result a very interesting case of synchronization between the coupled autonomous circuits of this network was revealed. According, to the chosen sets of circuits' initial conditions the system could be in two different dynamic states. When the autonomous circuits started from the same basin of attraction, the system of the coupled autonomous circuits under the influence of the stimulation of the Lacy circuit resulted in a complete chaotic synchronization state. However, when the autonomous circuits started from different basins of attraction, the system showed a totally new type of synchronization. In this case, which was called partial synchronization of autonomous circuits, only part of the corresponded electric variables of these circuits are synchronized to each other, while the state variables remain unsynchronized.

As a future work, the experimental observation of the synchronization phenomena that were the subject of this work and especially of the new observed case of partial synchronization will be studied. Also, the confirmation of existence of this new type of synchronization in the case of 
using other neuronal circuits, instead of Lacy circuits, is also important for the understanding of phenomena related to the nature of neuronal systems.

\section{References}

1. Pikovsky, M. Rosenblum, and J. Kurths, Synchronization: A universal concept in nonlinear sciences, Cambridge University Press, Cambridge, (2003).

2. E. Mosekilde, Y. Maistrenko, and D. Postnov, Chaotic synchronization: Applications to living systems, World Scientific, Singapore, (2002).

3. B. Nana, P. Woafo, and S. Domngang, Commun. Nonlinear Sci. Numer. Simul. 14, 2266 (2009).

4. Ch.K Volos, I.M. Kyprianidis, and I.N. Stouboulos, WSEAS Trans. Circ. Syst. 5, 1654 (2006).

5. H. Fujisaka and T. Yamada, Prog. Theory Phys. 69, 32 (1983).

6. A.S. Pikovsky, Z. Phys. B - Condensed Matter 55, 149 (1984).

7. L.M. Pecora and T.L. Carroll, Phys. Rev. Lett. 64, 821 (1990).

8. G.I. Dykman, P.S. Landa, and I. Neymark, Chaos Solit. Fract. 1, 339 (1991).

9. M.G. Rosenblum, A.S. Pikovsky, J. Kurths, Phys. Rev. Lett. 78, 4193 (1997).

10. N.F. Rulkov, M.M. Sushchik, L.S. Tsimring, and H.D.I. Abarbanel, Phys. Rev. E. 51, 980 (1995).
11. R. Mainieri and J. Rehacek, Phys. Rev. Lett. 82, 3042 (1999).

12. H.U. Voss, Phys. Rev. E 61, 5115 (2000).

13. G.H. Li, Chaos Solit. Fract. 40, 1076 (2009).

14. Ch.K. Volos, I.M. Kyprianidis, and I.N. Stouboulos, Int. J. Bifurc. Chaos 21, 2357 (2011).

15. Ch.K. Volos, I.M. Kyprianidis, and I.N. Stouboulos, Commun. Nonlinear Sci. Numer. Simulat. 16, 3356 (2011).

16. I.M. Kyprianidis and I.N. Stouboulos, Chaos Solit. Fract. 17, 317 (2003).

17. Ch.K. Volos, I.M. Kyprianidis, and I.N. Stouboulos, Int. J. Circuits, Systems and Signal Processing 1, 274 (2007).

18. L.Y. Cao and Y.C. Lai, Phys. Rev. 58, 382 (1998).

19. J.G. Lacy, Int. J. Bifurcat. Chaos 6, 2097 (1996).

20. L.A.B. Torres and L. Aguirre, Electronics Letters 36, 1915 (2000).

21. L. Fortuna, M. Frasca, and M.G. Xibilia, Chua's circuit implementations: Yesterday, Today and Tomorrow, World Scientific, Singapore, (2009). 